How far this may apply to moist agents of any description can only be determined by careful and continued study. If we are to reason from analogy or to act on the hints that we gain from the study of climatic treatment, we must primarily conclude that cases that should do well in warm, moist atmos- ! pheres of southern climates will do well with antiseptic vapors and spray; while those cases that should show improvement in cold, dry climates would best respond to dry dehydrating agents. The range of medication being so thoroughly under our control, it must require as good judgment and discrimination as by internal medication. I have found a hint in treating some cases, by changing as much as possible the character of the air from that in which the disease developed, and I have further found preliminary course of cabinet treatment to materially aid me in the selection of climate for individual cases. While the rapid improvement that has super vened upon a climatic change, after a preliminary course of pneumatic differential treatment may be ascribed to the effectual expansion thereby induced, I am sure I am warranted in my conclusion that a measure of the benefit is due to thorough disinfec. tion of the lungs and the evidence gained of the patient's tolerance of moist or dry air, as the case may be.

I will mention a few of the most important agents that I have thus far used: Dry air, this can be easily furnished by attaching an $U$ tube to the breathing faucet, and packing the tube with pumice stone and pure sulphuric acid; or absorbent cotton borated or mercurialized may be loosely packed in the tube.

The bichloride of mercury in solution of a strength of $\frac{1}{50} \pi$ to $\frac{1}{20} \pi^{\circ}$.

Lugol's solution 10 to 50 per cent.

Creosote 10 to 50 per cent.

Pure carbolic acid to to 50 per cent.

In using the last two agents I have projected the spray with the "Semple Inhaler," using equal parts of pure glycerine and water as diluents.

Oxygen gas and the per-oxide of hydrogen may be appropriately applied. I am now using C. P. glycerine as a de hydrating and disinfectant spray.

As emollient and sedative applications I have used morphine, cocaine, atropia, stramonium, and a weak emulsion of peppermint oil.

It is unnecessary to make further reference to special agents. The list is as extensive as the materia medica, and as in other departments of medicine, each practitioner will find certain agents to behave better in his hands than in those whom he seeks to instruct, yet as there is probably but one best way to do everything, it is equally probable that there is but one best bacillicide and one best opportunity for its perfect work.

450 Classon Avenue, Brooklyn, N. Y., April 26, 1887.

\section{BERGEON'S METHOD OF TREATING PHTHISIS BY GASEOUS ENEMATA.}

Read before the Chicago Medical Society, April 18,1887 . BY FRANCIS J. CRANE, M.D.,

\author{
OF CHICAGO, ILL.
}

In January last, the $N . Y$. Medical Record contained an article headed "A New Treatment of Phthisis," which gives a brief outline of Dr. Bergeon's method of administering carbon dioxide mixed with sulphuretted hydrogen gas, and referred to an article published in the British Medical Journal, of December 18,1886 . This described in full the apparatus and mode of using it, and stated they had been procuring beneficial results by the method, and that Professor Cornil, of Paris, had also become an enthusiastic supporter of it. This led me to write to Dr. Bergeon, and in the course of my correspondence, he presented me with an apparatus, as well as with the treatise of Dr. Morel read before the French Academy last June, and also one by Professor Cormil. These are so exhaustive that I have embodied in this paper only the essence of both, as follows:

New treatment of the diseases of Respiratory Organs and Septicemia by the means of Gascous Enemata according to M. Bergeon's Method. By Dr. V. Morel. It would seem from the statements of physicians who have tried either to prevent the development or the proliferation of the bacillus, or to destroy it, that it is the one thing which we have to overcome in the treatment of phthisis. In reasoning by analogy with other contagious diseases, as, for instance, cholera and hydrophobia, it might be urged that the bacillus is not the immediate cause of the morbid phenomena of tuberculous affections. It is known that, aside from miliary tubercles, which invade the lungs and are the cause of the patients' symptoms, the gravest phenomena of phthisis are due to the septicæmia, which poisons the patient, and is caused by the suppuration of the tubercles, which, brought in contact with air, undergo putrefaction and are absorbed into the system. The bacillus' work, then, by producing lesions of texture, which become fatal to the organism by rapidly destroying or, by undergoing softening and absorption, produces septicamia. To use Darembourg's expression: "The bacillus is nothing, but septicæmia is everything."

In acknowledging that it is not necessary to ascribe to the microbe all of the morbid phenomena of phthisis, it is not less true that its presence is to the organism an incessant and real danger, and that consequently, in endeavoring to discover a remedy for the lesions which it has produced, it is necessary to destroy it, or at least to neutralize its action. For this purpose such agents as sulphuret of hydrogen, bisulphide of carbon, and other antiseptics mixed with pure carbonic acid, are employed.

Principles of Bergeon's Method.-The first mode of treatment thought of consisted in applying by inhalation some substances having parasiticidal properties. It is known that antiseptic substances are poisonous when introduced into the arterial system, either directly or by inhalation. Claude Bernard has 
shown that poisonous gases introduced into the arterial system through the lungs produce toxic effects almost instantaneously. Besides, the antiseptic substances have an irritating action, and this action, operating on diseased lungs, will only increase the existing lesions, while their unpleasant odor aroused a refusal in the patients to their use; this is probably the reason why so little success has been obtained by inhalation in the treatment of phthisis, and is conclusive proof that the introduction of antiseptics by the stomach is preferable, for $\mathrm{Cl}$. Bernard has shown that when a poisonous or medicinal substance is introduced into an organ distant from the arterial system; into the digestive tract, for instance, it cannot enter the arterial system, because it is expelled before reaching it. It must pass through the portal system, the hepatic veins and the pulmonary texture, there to be exhaled, or it can be expelled in the liver with the bile.

It is well established by experiments that the introduction of poisonous substances in to the digestive tube may be done without danger by taking certain precautions, of which the most important consist in not injecting too large quantities at once, and not injecting more before the first has been completely eliminated. What avenue ought to be chosen? The stomach or the rectum? In both cases, the medicament will have to pass through the portal vein, the liver, the hepatic vein, the right heart and pulmonary arteries, but we think the rectal way is preferable, for the patient cannot take a dislike to the antiseptic substances on account of their disagreeable odors.

Gaseous Enemata in the Therapeutics of Respira tory Organs, Pulmonary Phthisis, Asthma, Whooping-cough, Bronchitis, Bronchiectasis, Bronchorrhoea, Pulmonary Catarrh. By M. Cornil. ${ }^{\text {The principle }}$ of the action of gaseous infections and of their rapid elimination by the lung, has been given by $\mathrm{Cl}$. Bernard. He showed that when sulphuretted hydrogen is injected into the rectum of an animal, the gas is expelled by the lungs; he proved that we can so inject it in almost unlimited quantities, without causing harm; whereas its introduction by inhalation rapidly occasions grave accidents and the animal's death. However, to introduce sulphurous hydro. gen, or any other gas or vapor, into the economy per rectum, for the purpose of destroying the microorganisms which exist in a number of diseases, it was necessary to find a gaseous vehicle, inoffensive to the economy, and easily tolerated by the bowel.

Carbonic acid gas admirably answers the purpose; it is very easily borne by the colon, rapidly absorbed and afterward expelled by the lung, with the medicinal gas which it holds. This gas itself, in all probability, plays a very important part in this new treatment of pulmonary diseases.

Dr. Bergeon, who inaugurated this method, published a few months ago the first results obtained in the treatment of pulmonary phthisis by this method. Physicians of Lyons, Paris, Geneva and Marseilles who have treated phthisis by the method, have generally obtained a very rapid disappearance of the phe-

1 See The Journal, January 15, 1887, p. 69. nomena of pulmonary suppuration, and a progress towards a state of health with all the signs of cure.

Concerning the patients I have treated by this method, I can now assert that the results I predicted three months ago have been achieved. The patients that I considered cured have no more expectoration, and give on auscultation stethoscopic signs which denote the presence of quiescent cavities, or cicatrized lesions. Some of these patients have been obliged to return to a life of labur; nevertheless their respiratory organs have stood the test, and the amelioration obtained has been permanent.

While many patients whom the expectoration once so exhausted, now have only 3 or 4 grams of sputum a day, at the beginning of the treatment it was from 250 to 300 grams. We have found bacilli, it is true, in the sputa of these patients; yet it remains to be discovered whether these bacilii which continue to exist after the return to health have kept their furctional activity or not. Whatever may be the mode of action of carbonic acid introduced by intestinal absorption in the venousblood and afterward expelled by the lung, it can be said from the observation of patients, that this gas, filled with proper medicinal substances, greatly modifies the respiratory function, and makes the hæmatosis more complete and easy. It gives a sensation of well-being, followed by an increase of strength and weight, a diminution of fever and night sweats.

The following precautions must be observed in giving this treatment: $\quad$. The $\mathrm{CO}^{2}$ ought to be as pure as possible, so as not to inflame the bowel. That obtained by the reaction of dilute sulphuric acid on the bicarbonate of soda has always been perfectly absorbed by the bowel without producing any toxic effect.

2. The gas should be collected in a receiver from which the air has been expelled.

3. Make the injections just before a meal, or at least three hours after, and never when the patient is weary. It is necessary to be very cautious in experimenting with other medicinal substances, for if, although the sulphuretted hydrogen is inoffensive, other agents, as turpentine, chloral, ammoniæ, iodine, bromine, ether, etc., may not be, and might be the cause of an inflammation of the intestinal mucous membrane.

It is not necessary that the dose be large; by injecting twice a day 4 or 5 litres of carbonic acid gas passed through 500 grams of sulphur water, we rapidly notice the disappearance of all the phenomena of pulmonary suppuration, either in its acute or chronical state.

Bergeon's method has been successfully experimented with by Dr. Chantamesse, in his service at St. Antoine Hospital, during the months of August, September and October. The following are his results: "Two patients brought to the hospital suffering with violent attacks of asthma were, half an hour after the injection with sulfo-carbon vapors, entirely relieved of the dyspnœa. The treatment having been continued for a few days, the breathing was relieved, and the attacks were not repeated during the time they remained." Nine patients giving general 
and local signs of pulmonary tuberculosis, with tubercular bacilli in the sputa, have obtained very great amelioration from this treatment. The increase of weight has been rapid, one pound and sometimes as much as 35 oz. a week; cough and expectoration have largely ceased. We always find the bacilli in the sputum, however. These patients have been under treatment for one month and a half. One of them has increased nine pounds in weight.

I have used this treatment with four cases; two of phthisis, one of intussusception of the bowel and one of spasmodic croup. With the croup and intussusception it operated like a charm, overcoming both almost instantly. In the case of croup, I used the bisulphide of carbon, and in half an hour the little patient was sleeping, apparently as well as ever.

Case I.-Mr. W., aged 26 . Two sisters and a brother died of phthisis; he had been treating with various physicians and changing climate (having been to Colorado twice) for over three years. The right lung was nearly useless, as it contained a cavity corresponding to nearly if not quite half of its original capacity. Nowhere on this side could vesicular respiration be heard, while the left apex likewise yielded unmistakable signs of disease. There were œedema of the feet, incessant cough, broken sleep, watery stools and ravenous appetite, although he could not retain anything on the stomach; temperature ${ }^{2} 2^{\circ} \mathrm{F}$. After the first injection of bisulphide of carbon, given in the evening, he slept well for three hours and was bothered very little with cough, but on rising in the morning, to use his own words, came nearly strangling for want of a cough, which he finally got, and expectorated a pint with the one paroxysm. I then used the sulphuretted hydrogen water and he improved very fast; in one week he had a normal temperature; night sweats almost entirely stopped, expectoration was much less, and he was able to wear his shoes, which he had not been able to do for over six weeks. Unfortunately, however, at the latter part of the second week he ventured out in one of our rainy March days, took cold, and his death, two days later, cut short the record of what might have proven almost a miracle.

Case 3.-Mrs. W., aged 34, widow, having lost her mother and older sister from phthisis, applied to me for some heart trouble. Complained of a dizzy sensation on rising from a recumbent position; feet swollen some, hectic flush, considerable dyspncea, slight cough with no expectoration. Diagnosis: Incipient phthisis with heart complication? She had noticed, also, for about a week some night sweats, which did not last, however, after the second administration of gas. She improved so rapidly that she only made seven visits in all, and pronounced herself cured. There is, however, no doubt but she will have a return of symptoms upon the slightest provocation.

This comprises all my experience, but these are facts, and facts are stubborn things to deal with. In

regard to the best mineral water, I wish to say that, after trying the Lafayette, Ind., the Blue Lick, Ky., and the Ypsilanti, Mich., mineral waters, I am satisfied that the Ypsilanti mineral water is just what we want. It contains 20 cubic inches of gas to the gallon, and is so strongly impregnated with it that I use it over the second time by having solid rubber corks to replace the perforated ones when I have got through using the apparatus. Mr. St. Clair, President of the Company at 88 Randolph St., has kindly furnished a case of I2 quarts of the water, which I have forwarded to Dr. Bergeon, in hopes that it will compare favorably with the Eaux Bonnes water which he is using. I have had an apparatus made by E. H. Sargent, of Chicago, which I think takes the place of Morel's very well, differing from it only in point of cheapness, costing but a little more than one-half the former.

The true place of this mode of treatment cannot be established until the experience of careful observers has been given us, years hence. I wish, therefore, to urge the profession to investigate the matter
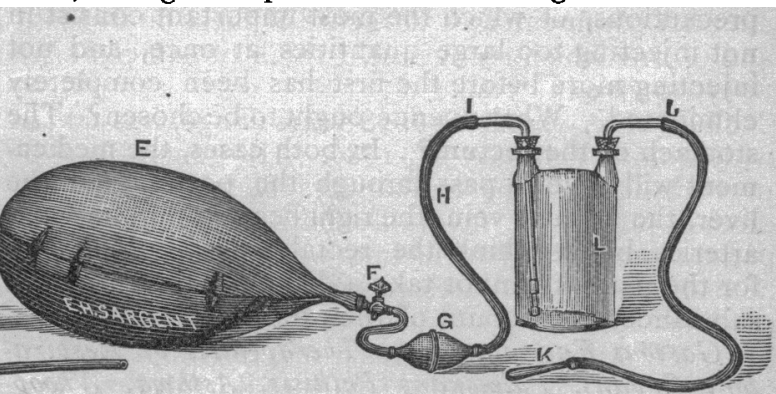
fairly, since time, I am confident, will prove that Dr. Bergeon has been one of the greatest benefactors of the age.

\section{ras}

The epidemics of mumps which are of frequent occurrence in almost every community, present some points of interest to which it may be well to refer. But little attention seems to be given in medical literature to the consideration of this disease. Aitken, in his "Science and Practice of Medicine," three volumes of over 3,000 pages, devotes one page to its consideration; and that monument to the progress of medical science, Pepper's "System of Medicine," containing about 5,500 pages, devotes less than six pages to the subject; J. Lewis Smith, in his work on the "Diseases of Infancy and Childhood," of over 800 pages, devotes two pages to this subject; and yet it is one of our most widely extended and frequently recurring epidemics.

Mumps, as it prevails under the usual conditions, does not possess a high degree of importance, even 